\title{
First report of reference guided genome assembly of Black Bengal goat (Capra hircus)
}

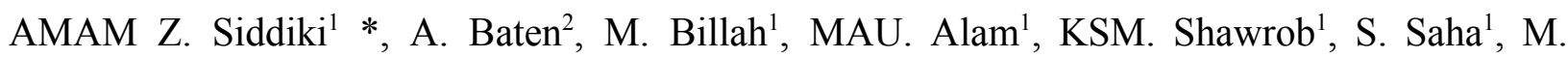
Chowdhury ${ }^{1,6}$, A.H. Rahman ${ }^{3}$, M. Stear ${ }^{4}$, G. Miah ${ }^{5}$, M. Kumkum¹, M.A. Hossain', AKM. Mollah $^{6}$, M.S. Islam ${ }^{1}$, MKI. Khan ${ }^{5}$

${ }^{1}$ Genomics Research Group, Faculty of Veterinary Medicine, Chittagong Veterinary and Animal Sciences University (CVASU), Chittagong, 4225, Bangladesh;

${ }^{2}$ AgResearch, Palmerston North 4410, Private Bag 11008, New Zealand;

${ }^{3}$ Department of Computer Science and Engineering, Bangladesh University of Engineering and Technology (BUET), Dhaka 1000, Bangladesh;

${ }^{4}$ AgriBio, Department of Animal, Plant and Soil Sciences, School of Life Sciences, La Trobe University, Bundoora, Victoria 3083, Australia;

${ }^{5}$ Department of Genetics and Animal Breeding, Faculty of Veterinary Medicine, Chittagong Veterinary and Animal Sciences University (CVASU), Chittagong, Bangladesh;

${ }^{6}$ Department of Biological Sciences, Asian University for Women (AUW), Chittagong, Bangladesh.

\section{Corresponding Author}

*AMAM Zonaed Siddiki DVM, MS, PhD

Address: Department of Pathology and Parasitology, Chittagong Veterinary and Animal Sciences University (CVASU), Khulshi, Chittagong-4202, Bangladesh

Mobile: 0088-01717718884

Email: zsiddiki@gmail.com

\section{zsiddiki@,cvasu.ac.bd}




\section{Abstract \\ Objectives}

Black Bengal goat (Capra hircus), a member of the Bovidae family with the unique traits of high prolificacy, skin quality and low demand for food is the most socioeconomically significant goat breed in Bangladesh. Furthermore, the aptitude of adaptation and disease resistance capacity of it is highly notable which makes its whole genome information an area of research interest.

\section{Data description}

The genomic DNA of local (Chittagong, Bangladesh) healthy Black Bengal goat (Capra hircus) was extracted and then sequenced. The de novo assembly and structural annotations are being presented here. Sequencing was done using Illumina sequencing platform and the draft genome assembled is about $3.04 \mathrm{~Gb} .26458$ Genes were annotated using Maker gene annotations tool which predicted BUSCO Gene models. Universal Single Copy Orthologs refer 82.5\% completeness of the assembled genome.

\section{Keywords}

Black Bengal goat, Capra hircus, Whole Genome sequence, Assembly, Annotations 


\section{Objective}

Black Bengal goat (BBG) belongs to the Bovidae family and found throughout Bangladesh, West Bengal, Bihar, and Orissa regions of northeastern India. It is estimated that more than $90 \%$ of the goat population in Bangladesh comprised the Black Bengal, the remainder being Jamunapari and their crosses [1]. Higher prolificacy, fertility, resistance against common diseases, adaptability to the adverse environmental condition, early maturity, seasonality and superiority in the litter size are some of the outstanding features of BBG. Besides, it produces excellent quality flavored, tender and delicious meat with low intramuscular fat and fine skin of extraordinary quality for which there is tremendous demand all over the world [1,2]. Moreover, it plays a vital role in the economy of Bangladesh by contributing $1.66 \%$ of the GDP (Gross Domestic Product) (DLS, 2017).

Fortunately, the market demand of Black Bengal goat is emerging. This gives breeders of original/rare breeds an opportunity to expand the stock and preserve its genetic diversity. One of the primary goals in managing goat populations is to maintain high-level genetic diversity and low-level inbreeding. To estimate the future breeding potential of a goat breed, it is necessary to characterize the genetic structure and evaluate the level of genetic diversity within the breed. Moreover, a long term genetic approach can be used to improve the spectacular economic characteristics of BBG [3].

Therefore, the genetic characterization of the entire BBG genome is essential in characterizing its economic traits as well as adaptive capability. With the availability of whole genome sequence, the targeted areas for genetic improvements are now: goat prolificacy, growth rate, meat quality, 
skin quality, disease resistance, and survivability. A complete and accurate reference to the goat genome is an essential component of advanced genomic selection of product characteristics.

\section{Data description}

At first, healthy Black Bengal Goats (BBG) without known genetic diseases were selected for blood collection. Genomic DNA from each animal was isolated from the EDTA-blood, using the Addprep genomic DNA extraction kit (South Korea) (detailed methodology in Data File 1, Table 1). The quality and quantity of the DNA were assessed by the Qubit fluorometer (Invitrogen, Carlsbad, CA, USA) and Infinite F200 microplate reader (TECAN), according to the manufacturer's instruction. The status of the DNA was visually inspected by $0.8 \%$ agarose gel electrophoresis. Purified genomic DNA was sent for library preparation (detailed methodology in Data File 1, Table1) and WGS sequencing at Beijing Genomic Institute (Beijing, China). A total of $40 \mathrm{~Gb}$ (14-fold) of subread bases with a read length of $150 \mathrm{bp}$ were generated using next-generation sequencing (NGS) technology on an Illumina HiSeq 2500 platform (detailed methodology in Data File 1, Table 1).

The quality of the reads was checked by using FastQC [4] and compared with the reference genome (San Clemente breed) ARS1 (GCA_001704415.1) which significantly improved the quality and continuity of the genome. As a result, the $\mathrm{N} 50$ increased from 87,277,232 $\mathrm{Kb}$ (kilobase pair) to $102,339,471 \mathrm{~Kb}$ and the total number of scaffold decreased from 29,907 to 3,972. Subsequently, for de novo assembly we used ABySS v. 2.1.5 assembler [5]. Furthermore, by using ABACAS v.1.3.1 and comparing with the reference genome; arranging, ordering, and orientation of the assembled genome was done [6]. The genome assembly data has been deposited in the NCBI GenBank under the Accession numbers GCA_001704415.1(Data file 2; 
Table 1). The final assembled genome size of Capra hircus is now 3.04Gb (Gigabase pair) 82.5\% completeness was revealed by BUSCO [7] analysis. Moreover, a considerably better N50 size and lower scaffolds number suggested a higher quality of the genome. The whole genome sequence data has been submitted in the NCBI GenBank under the Accession number SMSF01000001-SMSF01003972 (Data file 3; Table 1).

Consequently, to do the structural annotations Maker ver 3.0 pipeline was applied [8]. In the genome, $41.77 \%$ of GC content was observed. The latest version of the repbase database identified $31.85 \%$ repeat elements by using Repeatmasker [9]. After applying MAKER gene annotation pipeline 26458 genes were predicted which was based on both Denovo and reference guided. Finally, InterProScan [10] predicted 12589 genes out of 26458 genes, and 8173 genes have at least one GO term.

Table 1: Overview of data files/data sets.

\begin{tabular}{|c|c|c|c|}
\hline Label & Name of data file/data set & $\begin{array}{l}\text { File types } \\
\text { (file extension) }\end{array}$ & Data repository and identifier (DOI or accession number) \\
\hline Data file 1 & $\begin{array}{l}\text { DNA isolation and library } \\
\text { preparation methodology }\end{array}$ & docs file & https://doi.org/10.6084/m9.figshare.7965080.v1 \\
\hline Data file 2 & $\begin{array}{l}\text { Whole genome assembly } \\
\text { data }\end{array}$ & FASTA & $\begin{array}{l}\text { NCBI GeneBank (Accession numbers: GCA_0043616 75.1) } \\
\text { (http://identifiers.org/ncbi/insdc.gca:GCA_00436167 5.1) }\end{array}$ \\
\hline Data set 3 & Whole genome sequence & FASTA & $\begin{array}{l}\text { NCBI GeneBank (Accession numbers SMSF01000001- } \\
\text { SMSF01003972) ( } \underline{\text { http://identifiers.org/ncbi/insdc:SMSF0 }} \\
\underline{0000000.1)}\end{array}$ \\
\hline
\end{tabular}




\title{
Limitations
}

The number of unassembled regions in the genome is 3943.

\begin{abstract}
Abbreviations
BBG: Black Bengal Goat; GDP: Gross Domestic Production; EDTA: Ethylene diamine tetra-acetic acid; DNA: Deoxyribonucleic acid; WGS: Whole Genome Sequencing ; BUSCO: Benchmarking Universal Single-Copy Orthologs; ABACAS: Algorithm-based automatic contiguation of assembled sequences; Gb: Gigabase pair; Mb: Megabase pair; Kb: Kilobase; bp: Base pair; GO: Gene ontology;; SNAP: Semi-HMM-based Nucleic Acid Parser.
\end{abstract}

\section{Declarations}

\section{Ethics approval and consent to participate}

The experiments discussed in this investigation were approved by the Institute Review Committee of Chattogram Veterinary and Animal Sciences University

\section{Consent for publication}

Not applicable 


\section{Availability of data material}

The genome sequence information has been accessible at DDBJ/ENA/GenBank under the Accession numbers SMSF01000001-SMSF01003972 and the assembled genome at GCA_001704415.1. The version reported in this paper is the first version, SMSF00000000.1

\section{Competing interests}

The authors declare that they have no competing interests

\section{Funding}

This material was based upon work supported by the UGC funded projects underway at Chittagong Veterinary and Animal Sciences University (CVASU).

\section{Authors' contributions}

All authors contributed equally

\section{Acknowledgments}

Authors concede the support of Beijing Genomic Institute for the sequencing service and that of Southern Cross University, Lismore, Australia for the computational support.

\section{References}

1. Husain SS, 1993. A Study on the productive performance and genetic potentials of Black Bengal goats. 
2. Islam M, TN Nahar, and S Hoq, 1991. Prospect of goat production in Bangladesh. Asian Livestock Livest. 16: 85-95

3. Faruque S, Chowdhury SA, Siddiquee NU, Afroz MA. Performance and genetic parameters of economically important traits of Black Bengal goat. Journal of the Bangladesh Agricultural University. 2010;8(1):67-78.

4. FastQC: FastQC program. https://www.bioinformatics.babraham.ac.uk/projects/fastqc/. Accessed 12 Jan 2017

5. Abyss Simpson JT, Wong K, Jackman SD, Schein JE, Jones SJ, Birol I. ABySS: a parallel assembler for short read sequence data. Genome research. 2009 Jun $1 ; 19(6): 1117-23$

6. ABACASAssefa S, Keane TM, Otto TD, Newbold C, Berriman M. ABACAS: algorithm-based automatic contiguation of assembled sequences. Bioinformatics. 2009 Jun 3;25(15):1968-9.

7. BUSCO: Simão FA, Waterhouse RM, Ioannidis P, Kriventseva EV, Zdobnov EM. BUSCO: assessing genome assembly and annotation completeness with single-copy orthologs. Bioinformatics. 2015 Oct 1;31(19):3210-2.

8. Maker: Cantarel BL, Korf I, Robb SM, Parra G, Ross E, Moore B, Holt C, Alvarado AS, Yandell M. MAKER: an easy-to-use annotation pipeline designed for emerging model organism genomes. Genome research. 2008 Jan 1;18(1):188-96.

9. Repeat Masker: Smit A, Hubley R, Green P. RepeatMasker open-4.0. 2013-2015. Seattle, WA, USA: Institute for Systems Biology; 2015. http://www.repeatmasker.org/faq.html 
10. Quevillon E, Silventoinen V, Pillai S, Harte N, Mulder N, Apweiler R, Lopez R.

InterProScan: protein domains identifier. Nucleic acids research. 2005 Jul 1;33(suppl_2):W116-20. 